\begin{tabular}{c} 
Volume and Issues Obtainable at Center for Sustainability Research and Consultancy \\
Journal of Business and Social Review in Emerging Economies \\
ISSN: $2519-089 X$ \& ISSN (E): 2519-0326 \\
Volume 7: Issue 2 June 2021 \\
CSR \\
Jofit \\
Journal homepage: $\underline{\text { www.publishing.globalcsrc.org/jbsee }}$ \\
\hline
\end{tabular}

\title{
Improving Customers Satisfaction through Significance of Technical Attribute in QFD Studies
}

*Zafar Iqbal, Department of Statistics, The Islamia University of Bahawalpur, Pakistan Lubna Shoukat, Department of Educational Training, The Islamia University of Bahawalpur, Pakistan

Muhammad Waheed, Department of Educational Research and Evaluation, The Islamia University of Bahawalpur, Pakistan

Muhammad Rajab, Department of Statistics, The Islamia University of Bahawalpur, Pakistan

*Corresponding author's email: zafariqbal101@ hotmail.com

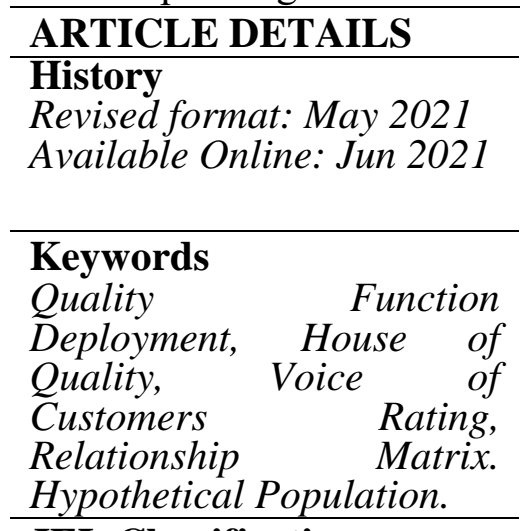

JEL Classification

M11, M12

$\begin{array}{llll}\text { ABSTRACT } & & & \\ \text { Purpose: Quality Function Deployment, (QFD) is a }\end{array}$
methodology which helps to satisfy customer requirements through the selection of appropriate Technical Attributes (TAs). The rationale of this article is to provide a method lending statistical support to the selection of TAs. The purpose is to determine the statistical significance of TAs through the derivation of associated significance (P) values. Design/Methodology/Approach: We demonstrate our methodology with reference to an original QFD case study aimed at improving the educational system in high schools in Pakistan; and then with five further published case studies obtained from literature. Mean weights of TAs are determined. Considering each TA mean weight to be a Test Statistic, a weighted matrix is generated from the VOCs' importance ratings, and ratings in the relationship matrix. Finally using R, $\mathrm{P}$-values for the means of original TAs are determined from the hypothetical population of means of TAs. Findings: Each TA's P-value evaluates its significance/insignificance in terms of distance from the grand mean. P-values indirectly set the prioritization of TAs. Implications/Originality/Value: The novel aspect of this study is extension of mean weights of TAs, to also provide $\mathrm{P}$-values for TAs. TAs with significant importance can be resolved on priority basis, while other can be fixed with appropriateness.

\section{OPEN $\bigcirc$ ACCESS}

(C) 2021 The authors, under a Creative Commons AttributionNonCommercial 4.0

\footnotetext{
Recommended citation: Iqbal, Z., Waheed, S., Waheed, M. and Rajab, M. (2021). Improving Customers Satisfaction through Significance of Technical Attribute in QFD Studies. Journal of Business and Social Review in Emerging Economies, 7 (2), 415-432.
} 


\section{Introduction}

Quality Function Deployment (QFD) was established by Japanese engineers and facilitated Japanese companies to improve their quality in the overseas markets successfully (Walker, 2002). It is a systematic approach towards the improvement of structures, features, requirements, qualities or utilities that give a product or service good or enhanced quality (Ahmad, 2006). QFD is a methodology for translating the Voice of the Customer (VOC) into suitable technical quality features that meet customer needs, wants and expectations. It also provides a source for product or service development and improvement (Aytac and Deniz, 2005). QFD is not only a methodological instrument but also a general approach that presents the resources for converting customer requirements in every phase of product/service improvement. QFD builds quality into a product by making certain that customer requirements are included into every phase of the improvement life cycle. Tortorella, et al. (2018) worked on to measure variation (variance of senior managers) and integrating variability into the policy deployment process.

Although QFD is well tried, tested and proven methodology, it has areas for potential improvement. Continuous improvement is a cornerstone principle of Quality Management, and therefore we should always seek new ways to improve such tools where possible. One area of potential weakness (and, therefore, of improvement) is in its lack of statistical significance testing in the prioritisation of Technical Attributes (TAs). Whilst calculated weightings are produced in order to rank and prioritise TAs, there are no associated P-values to enable practitioners to determine whether any TA's are significantly different from others, or whether observed variations between TAs are merely manifestations of random error (analogous to common cause variation). The separation of common cause from special cause variation, for the purpose of improved decision making, is a further cornerstone principle of quality management. Previous research by the present authors, Iqbal, et al. (2014) addressed this shortcoming through the introduction of a bootstrap re-sampling method to produce a theoretical distribution, and thereby obtain significance levels for the differences between pairs of TAs.

P-values are important in statistics as they represents the probability that a null hypothesis is incorrectly rejected (a Type I error is made). In the context of this paper, it is therefore a measure of risk of making an incorrect decision when selecting a TA as being more or less important than others. This equates to the risk that a QFD practitioner makes a false conclusion that a given TA is significantly different from the grand mean of all TA values, and then acts inappropriately on this conclusion. While in many cases this possible error will not be of practical importance, in certain critical or high value systems, correct prioritisation of TAs can be quite crucial.

The aim of this research article is, therefore, to improve the QFD methodology, by developing a methodology to determine P-values associated with the mean weights of TAs. The reason for selecting TA means (rather than other measures of central tendency) is because the ratings of the Relationship Matrix and VOC's, which play a vital role to compute P-values, are based on Likert scales with limited value ranges, and will not exhibit extreme values. Also no substantial skewedness is normally observed. Further details about the rating systems are given in the literature review section.

The developed methodology will make the TA selection statistically more reliable. Assigning Pvalues to TAs in any QFD application will provide practitioners, engineers, researchers, product developers, scientist etc., with a new way of setting priority rating of TAs for more critical applications. In the next section, a literature review is provided, starting with a brief introduction of QFD, then a review of various qualitative rating scales and methods that have been used to improve QFD methodology. This is followed by the methodology to determine TA P-values, and finally application of developed methodology to an empirical case study conducted by us and published (Iqbal, et al., 2020) in the field of education, and also on data from five published case 
studies.

\section{Literature Review}

\section{QFD, House of Quality Framework}

Figure 1 shows various matrices of the House of Quality (HOQ) used in QFD methodology. The QFD-HOQ always start with the VOCs. It integrates VOCs with some other matrices (TAs correlations, competitors' ratings, relationship matrix etc) and finally assimilates at the determination of TAs' weights. A brief detail of the matrices used in this process is given below.

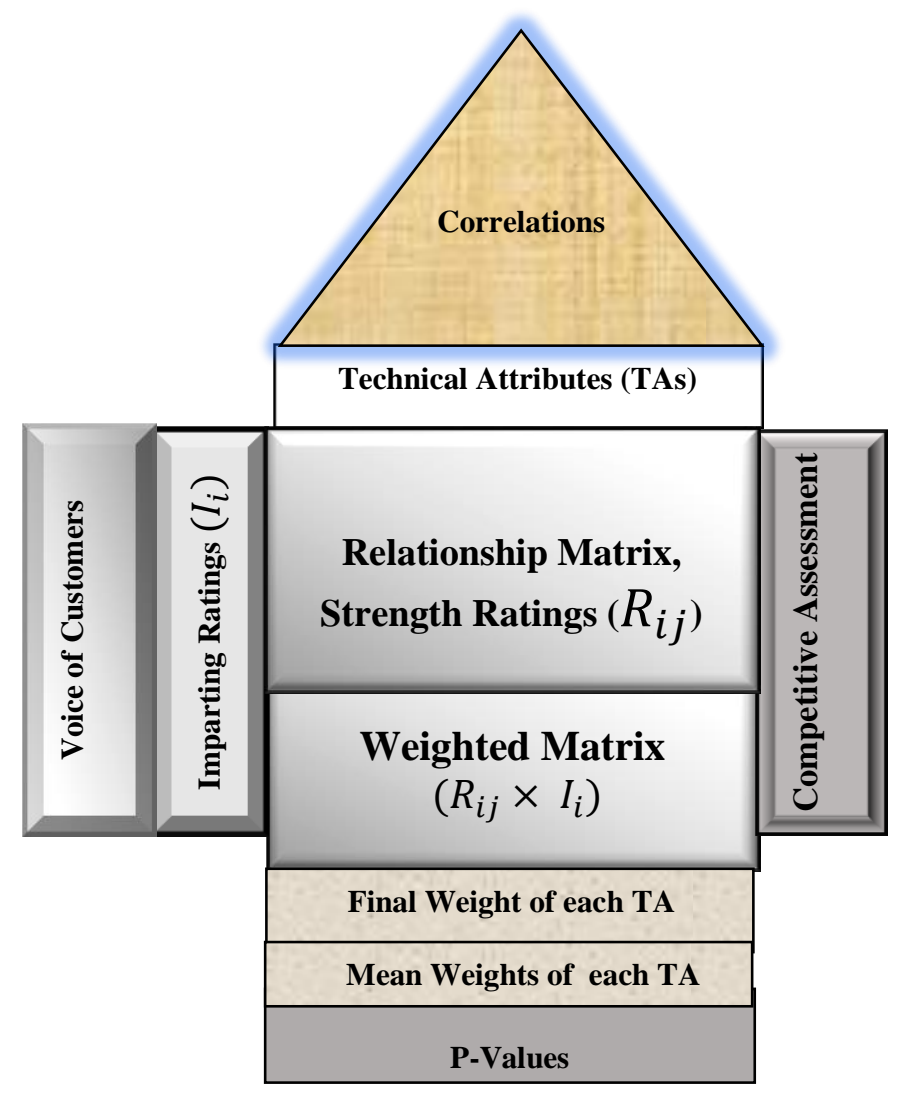

Figure 1: QFD, House of Quality

- Voice of Customers (VOCs): VOCs determination is the initial step in HOQ, and consists of determining, clarifying, and specifying the customer's needs, in their own voice.

- $\quad$ Technical Attributes (TAs): Technical Attributes are technical/engineering requirements required to satisfy VOCs. This step to identifying explicit product characteristics, features or attributes and presented how they will satisfy customer wants and needs.

- Interrelationship Matrix (also called Relationship Matrix): The main purpose of interrelationship matrix is to express the relationships between the customer's various, sometime conflicting, product requirements.

- Technical Correlation Matrix: This is frequently referred to as the 'Roof'. This matrix is used to express strength of relationships between the TAs. It shows how strong or weak correlations are between one TA and the remaining ones.

- Determination of Mean Weight of TAs: In order to compute mean weight of TAs, the first most common way is the determination of the final weights of TAs, which is the sum of the product of VOCs with each of the TA, (Iqbal, et al. 2014, Franceschini and Rossetto, 2002; Thakkar et al., 2006; Tan et al., 1998)

$$
F w_{j}=\sum_{i=1}^{r} R_{i j} \times I_{i} \quad i=1, \ldots, r \quad, j=i, \ldots, c
$$


Where,

$F w_{j}=$ Weights of $\mathrm{j}_{\mathrm{th}} \mathrm{TA}$

$R_{i j}=$ Strength of $\mathrm{j}_{\text {th }}$ TA with $\mathrm{i}_{\text {th }}$ VOC

$I_{i}=$ Customer importance rating

$\mathrm{i}=$ Number of VOCs

$\mathrm{j}=$ Number of TAs

In the present article, a new matrix is generated by multiplying each VOC rating with relationship matrix ratings, and this is called the Weighted Matrix, $W_{i j}$ i.e. $W_{i j}=R_{i j} \times I_{i}$

Here, $W_{i j}$ is the weighted strength of $\mathrm{j}_{\text {th }}$ TA with $\mathrm{i}_{\text {th }}$ VOC. Now, the mean weight of the weighted matrix is determined by using equation 2 .

$$
\bar{X}=\frac{\sum_{j=1}^{c} \sum_{i=1}^{r} R_{i j} \times I_{i}}{r \times c}
$$

The mean weight will be used to simulate new weighted matrices and each new weighted matrix mean will be used to determine a hypothetical population of means.

Technical attributes Priority Ratings: The final output of the HOQ is a set of final weights of TAs. The final weights are determined by using equation (1). The order of these TAs weights set the priority ratings, whereby TAs with higher weight have higher priority to resolve, and TAs with lower weight have lower priority to resolve. In order to explore new ways of interpreting the ranking of TAs, there is a need explore the factors to find all possible rankings and probability of the actual ranking among all possible rankings.

QFD practitioners have applied the QFD method in almost every field of business. For example, (Trappey et al., 1996) developed a formal QFD methodology for the retail industry and to build a computerized retail QFD system. (Lam and Zhao, 1998) expended an application of quality function deployment to improve the quality of teaching. Similarly, a hybrid methodology is introduced by (Wu and Lin, 2012) to develop an analytical model for enhancing the service quality of e-learning. Raharjo et al. (2007) applied the QFD and Analytic Hierarchy Process to Improve Higher Education Quality. Chin et al. (2001) elaborated a six-stage QFD approach based on an empirical study of the provision of the library and information services (LIS) in a technical organization in Hong Kong. Herrmann et al. (2006) conducted an empirical study on company performance by using quality function deployment. Vinodh and Chintha (2011) conducted a research study for enabling leanness in a manufacturing organization by using fuzzy Haber and Fargnoli, (2019) used QFD for prioritizing the health sector customer needs in the competitor matrix. Iqbal and Grigg (2020) focused on the enhancement of QFD based voice of customer's ratings by integrating the competitor matrix. Using competitors ratings they improved VOC's ratings.

QFD has also been applied to: e-Business planning which links both in strategic and operations management by (Tan et al., 2004); the software development process (Barnett and Raja, 1995); pultrusion machine design planning (Rahim and Baksh, 2003); enhancement of nursing home service quality by (Chang, 2006); the Design of a Lithium Battery by (Halbleib, 1993); application in the Hospitality Industry by (Paryani et al., 2010); service industry application by (Mazur, 1993); to rehabilitee services (Einspruch et al., 1996); improving soccer (Partovi and Corredoira, 2002) and the confectionery industry (Viaene and Januszewska, 1999). EstebanFerrer and Tricas (2012) recommended a practical framework for strategic quality management in law firms by applying the quality function deployment (QFD) principles to integrate the voice of the client into the law firms' resource enhancement process. Laosirihongthong et al., (2018) 
implemented Analytical Hierarchy Process, (AHP) with fuzzy logic to prioritise various performances combined with model of linear programming while Tusnial, et al. (2020) developed a decision-making model for supplier selection by integrating QFD and AHP.

Some researchers have worked on QFD methodology, in order to obtain more reliable and consistent results. Some improved the ordinal ratings used in its various matrices; some integrated other factors which may affect QFD results and while some joint other methodologies with QFD for better achievements of results. In the VOC matrix, although ordinal ratings, 1 to 3, 1 to 9,1 to 10 has been employed, most commonly used ratings are 1 to 5 (Olewnik and Lewis, 2008, Masui et al. 2003).

In the relationship matrix, the commonly used ratings for the strength of relationship between VOCs and TAs are 3-point, 5-point with different strength. For example, for 3-point, weak, medium and strong relationship, (Tan et al., 1998) used 1,3, 5, respectively; for weak, medium and strong relationship, (Jeong and Oh, 1998) used 1, 3, 10; while some used 1,3,9. Five-point ratings e.g 1,3,5,7,9 and 1,2,3,4,5 are also used to express, very weak, weak, medium, strong and very strong, relationship.

Finally we focus the main application of our research. The review further revealed that in the case of QFD for the improvements of the quality of products/services researchers, practitioners or engineers have mainly focused on the traditional method of finding final weights of TAs and then achieving these TAs according to their priority ratings. For example, Tan et al., (1998) used QFD in designing IT and focused on final rating of TAs. Stehn and Bergström (2002) integrated customer oriented design into ordered functional requirements (TAs) for the multi-story timber frame houses. Tan et. al., (2004) used QFD for e-Business planning, and comprehend e-Potentials and transform them into priorities e-Implementation. Maritan and Panizzolo (2009) identify business priorities through ranking of TAs. Andronikidiset al., (2009) explored various studies and acknowledge that the QFD method is an good methodology to recognise and evaluate the customers' expectations and requirements and, transform them into TAs whether it product, or a service or both.

Dror (2017) applied QFD method as a tool for ordered operation scheme of an individual organisation to achieve its business goals. Bulut et. al., (2018) used the multilayer QFD model for the service quality assessment of Kansai International Airport, Japan, which is capable of analysing the needs of all agents in order to find appropriate solutions. Sing and Rawani, (2018) prioritised the quality parameters of National Board of Accreditation, in order to improve the quality of higher education. Dror, (2019) wrote an article, entitled "Linking operation plans to business objectives using QFD”. He suggested competitive priorities to meet business objective for a furniture firm. Iqbal, et. al. (2014) made statistical comparison of final weights of QFD and suggested some TAs are equal in priority. It has been applied in the health industry as an an effective and appropriate service strategy, (Gambarovet al., 2017).

The literature review revealed no further methodological development for the selection of TAs. In the next section, we describe, how the TA weights can be determined statistically.

\section{Methodology: Testing the Significance of $\bar{X}_{j}$}

This paper provides an extension to a previous development by the authors, Iqbal, et al. (2014). We previously argued that an inferential statistical basis can be introduced via bootstrap sampling, and comparing each pair of TAs for significant difference. In this paper, we provide a variation on this theme, which is to test each TA average against the 'grand mean' of the TAs, and thereby establish whether any TA is significantly difference from that mean. This 
encompasses the concept of statistical thinking, a core principle within quality engineering (e.g. Snee, 1990) which urges quality practitioners to make decisions based on statistical evidence. Our proposed method proceeds as follows.

The two compulsory matrices ratings of the QFD methodology are VOCs' Importance Ratings and relationship matrix Strength Ratings, (Iqbal, et al. 2014). Commonly QFD practitioners integrate these two matrices and create another matrix by using the $R_{i j} \times P_{i}$ component of equation (1). This new matrix is named the Weighted Matrix, (WM, figure 1). Finally, WM is transformed into final weights of TAs by taking sum of each column of WM. Then the means $\bar{X}_{J}$ of these final TA weights are computed by dividing each final weight by the total number of VOCs $(r)$. Now considering mean of each TA as a test-statistic, under the null hypothesis that all the mean weights are same as population mean $\mu$. In order to test each of the means, because of ordinal rating used in all matrices of QFD, no assumptions about the population can be made, so there is a need to generate a hypothetical population. Then P-Values for each $\bar{X}_{J}$ can be generated. In order to generate hypothetical population, we assume the mean of $\bar{X}_{J}$ is equal to population mean $\mu$, and applied a parametric approach of generating samples, (Iqbal, et al. 2014) and generated a very large number of relationship matrix with mean $\mu$, for example, $\operatorname{rpois}(\mu, r \times c$ ), For each re-sampled relationship matrix, we generate WM, then final weights and a hypothetical population of mean weights, $\mu_{j}$ of each TAs are generated. Then we determine the P-value, (probability value) i.e $P\left(\bar{X}>\bar{x}_{\jmath} \mid H_{0}\right.$ is true) related with all Test-Statistics $\bar{X}_{j}$ of mean weights of TAs,. In this process, if the probability of our observed test-statistic is less than level of significance, traditionally called $\alpha$ on the hypothetical sampling distribution, then the $\bar{x}_{J}$ can be considered significant, representing that practitioners can priorities their action for more reliable achievement.

\section{Case Study: Application of the Develop Methodology}

A case study was conducted by the present researchers to improve education system in high schools, (Iqbal, et al., 2020). Details of Voice of Students, Selection of Technical Attributes, and all other steps until the determine of P-values are described in next section.

\section{Voice of Students}

As a first step of QFD framework, the following 'voices of students' were finalized with their importance ratings. These are translated verbatim from students responses in Urdu.

Table 1: List of Voice of Customers (VOCs) Criteria to Improve Quality of Education, (Iqbal, et al., 2020)

\begin{tabular}{|c|l|c|}
\hline Sr. No. & \multicolumn{1}{|c|}{ Voice of Students } & $\begin{array}{c}\text { Importance } \\
\text { Ratings }\end{array}$ \\
\hline 1 & Qualification and skills of teachers & 4.73 \\
\hline 2 & Limited students' strength in class & 3.96 \\
\hline 3 & Excursion trips/picnics & 3.55 \\
\hline 4 & Motivations & 4.54 \\
\hline 5 & Punctuality of teachers & 4.69 \\
\hline 6 & Special Place for studies & 4.18 \\
\hline 7 & Extra-Curricular Activities & 3.71 \\
\hline 8 & Teachers' role in academic performance & 4.54 \\
\hline 9 & Fix timing for studies & 4.65 \\
\hline 10 & All students treated equally by teachers & 4.73 \\
\hline 11 & Practical implementation by Models & 4.07 \\
\hline 12 & Monthly parents teacher meeting & 4.08 \\
\hline
\end{tabular}




\section{Technical Attributes}

After finalising the VoSs, practiced teachers, principals of schools, carrier and education consultants helped to finalized the Technical Attributes (TAs), Table 2, that can best resolve the VoSs. Following is the list of TAs that were finalized.

Table 2: List of Technical Attributes to Resolve Voice of Students, (Iqbal, et al., 2020)

\begin{tabular}{|c|c|c|c|c|c|c|c|c|c|c|c|c|c|}
\hline \multicolumn{14}{|c|}{ Technical Attributes (TAs) } \\
\hline 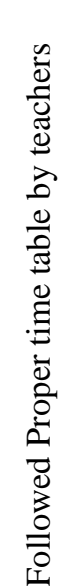 & 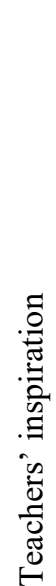 & 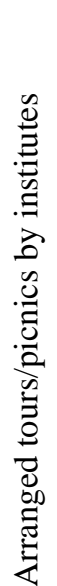 & 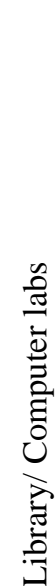 & 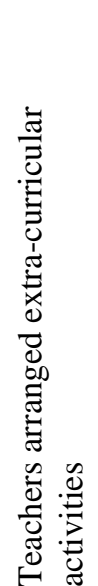 & 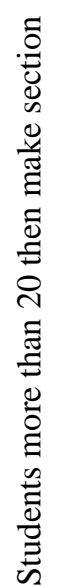 & 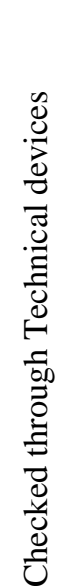 & 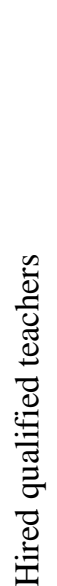 & 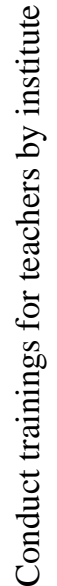 & 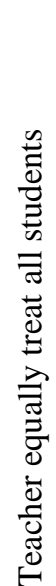 & 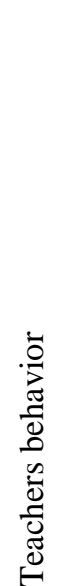 & 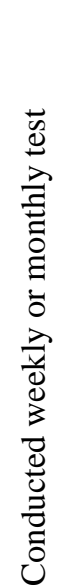 & 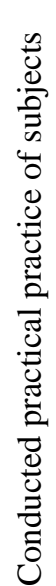 & 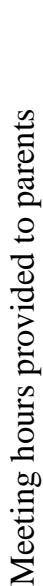 \\
\hline
\end{tabular}

\section{House of Quality for Students Requirements.}

Finally, House of Quality was developed, Table 3, which shows VoSs. TAs, Relationship Matrix, Final and Mean Weights, using equation 1 and 2 simultaneously.

Table 3: House of Quality, Showing VoSs, TAs, Relationship Matrix, Final Weights, Mean Weights. (Iqbal, et al., 2020)

\begin{tabular}{|c|c|c|c|c|c|c|c|c|c|c|c|c|c|c|c|}
\hline (TAs) & 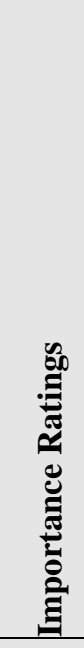 & 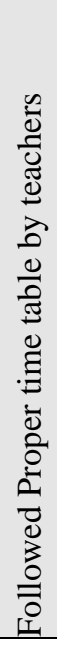 & 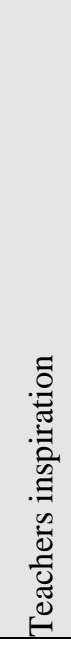 & 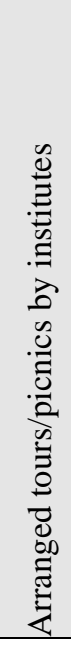 & 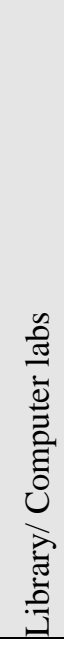 & 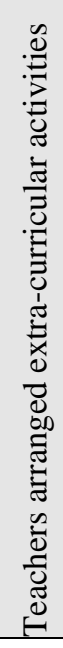 & 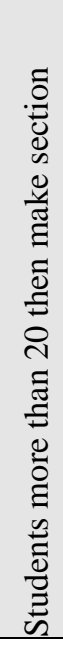 & 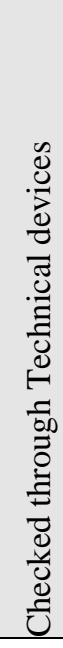 & 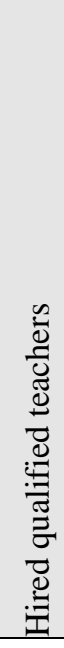 & 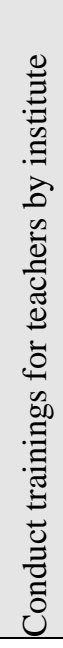 & 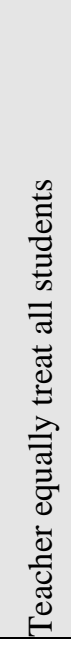 & 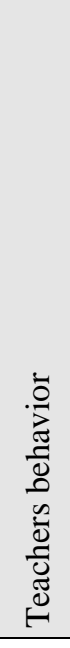 & 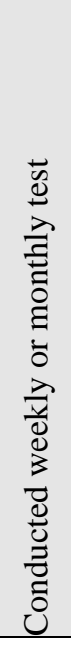 & 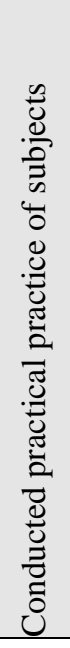 & 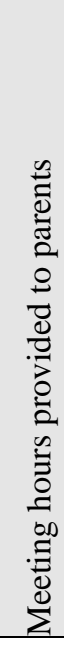 \\
\hline Qualification and skills of teachers & 4.73 & 0 & 0 & 0 & 0 & 0 & 0 & 0 & 7 & 6 & 0 & 0 & 0 & 0 & 0 \\
\hline Limited students strength in class & 3.96 & 0 & 0 & 0 & 0 & 0 & 5 & 0 & 0 & 0 & 0 & 0 & 0 & 0 & 0 \\
\hline Excursion tour/picnic & 3.55 & 0 & 0 & 6 & 0 & 5 & 0 & 0 & 0 & 3 & 0 & 0 & 0 & 3 & 0 \\
\hline Motivations & 4.54 & 0 & 0 & 0 & 0 & 0 & 0 & 0 & 0 & 0 & 0 & 6 & 0 & 0 & 0 \\
\hline Punctuality of teachers & 4.69 & 7 & 0 & 0 & 0 & 0 & 0 & 6 & 0 & 0 & 0 & 0 & 0 & 0 & 0 \\
\hline
\end{tabular}




\begin{tabular}{|c|c|c|c|c|c|c|c|c|c|c|c|c|c|c|c|}
\hline Special Place for studies & 4.18 & 0 & 0 & 0 & 6 & 0 & 0 & 0 & 0 & 0 & 0 & 0 & 0 & 0 & 0 \\
\hline Extra-Curricular Activities & 3.71 & 0 & 0 & 0 & 0 & 6 & 0 & 0 & 0 & 0 & 0 & 0 & 0 & 0 & 0 \\
\hline $\begin{array}{l}\text { Teachers' role in academic } \\
\text { performance }\end{array}$ & 4.54 & 0 & 5 & 0 & 0 & 0 & 0 & 0 & 0 & 0 & 0 & 5 & 6 & 0 & 0 \\
\hline Fix timing for studies & 4.65 & 5 & 0 & 0 & 0 & 0 & 0 & 6 & 0 & 0 & 0 & 0 & 0 & 0 & 0 \\
\hline $\begin{array}{l}\text { All students treated equally by } \\
\text { teachers }\end{array}$ & 4.73 & 0 & 0 & 0 & 0 & 0 & 0 & 0 & 0 & 0 & 6 & 5 & 0 & 0 & 0 \\
\hline $\begin{array}{l}\text { Practical implementation by } \\
\text { Models }\end{array}$ & 4.07 & 0 & 0 & 0 & 0 & 0 & 0 & 0 & 0 & 0 & 0 & 0 & 0 & 6 & 0 \\
\hline Monthly parents teacher meeting & 4.08 & 0 & 0 & 0 & 0 & 0 & 0 & 0 & 0 & 0 & 0 & 0 & 0 & 0 & 5 \\
\hline \multirow{12}{*}{\multicolumn{2}{|c|}{ Weighted Matrix }} & 22 & 0 & 0 & 0 & 0 & 0 & 28 & 0 & 0 & 0 & 0 & 0 & 0 & 0 \\
\hline & & 0 & 22 & 0 & 0 & 0 & 0 & 0 & 0 & 0 & 0 & 23 & 26 & 0 & 0 \\
\hline & & 0 & 0 & 22 & 0 & 18 & 0 & 0 & 0 & 12 & 0 & 0 & 0 & 11 & 0 \\
\hline & & 0 & 0 & 0 & 23 & 0 & 0 & 0 & 0 & 0 & 0 & 0 & 0 & 0 & 0 \\
\hline & & 0 & 0 & 0 & 0 & 23 & 0 & 0 & 0 & 0 & 0 & 0 & 0 & 0 & 0 \\
\hline & & 0 & 0 & 0 & 0 & 0 & 20 & 0 & 0 & 0 & 0 & 0 & 0 & 0 & 0 \\
\hline & & 33 & 0 & 0 & 0 & 0 & 0 & 29 & 0 & 0 & 0 & 0 & 0 & 0 & 0 \\
\hline & & 0 & 0 & 0 & 0 & 0 & 0 & 0 & 33 & 27 & 0 & 0 & 0 & 0 & 0 \\
\hline & & 0 & 0 & 0 & 0 & 0 & 0 & 0 & 0 & 0 & 30 & 24 & 0 & 0 & 0 \\
\hline & & 0 & 0 & 0 & 0 & 0 & 0 & 0 & 0 & 0 & 0 & 25 & 0 & 0 & 0 \\
\hline & & 0 & 0 & 0 & 0 & 0 & 0 & 0 & 0 & 0 & 0 & 0 & 0 & 23 & 0 \\
\hline & & 0 & 0 & 0 & 0 & 0 & 0 & 0 & 0 & 0 & 0 & 0 & 0 & 0 & 21 \\
\hline \multicolumn{2}{|l|}{ Final Weights } & $\begin{array}{l}\infty \\
\stackrel{\infty}{0} \\
\stackrel{0}{n}\end{array}$ & $\begin{array}{l}\stackrel{尺}{i} \\
\text { ה }\end{array}$ & 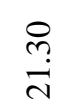 & $\begin{array}{l}\infty \\
\stackrel{d}{i}\end{array}$ & $\begin{array}{l}\overrightarrow{0} \\
\dot{q}\end{array}$ & $\begin{array}{l}\infty \\
\stackrel{\infty}{-}\end{array}$ & $\begin{array}{l}\text { J̦ } \\
\stackrel{0}{n}\end{array}$ & $=$ & $\begin{array}{l}\text { oे } \\
\text { ळे }\end{array}$ & $\begin{array}{l}\infty \\
\text { m } \\
\infty \\
\infty\end{array}$ & $\stackrel{n}{n}$ & 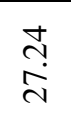 & $\begin{array}{l}\hat{0} \\
\dot{m}\end{array}$ & 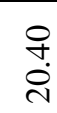 \\
\hline \multicolumn{2}{|l|}{ Mean of Final Weights } & $\hat{\sigma}$ & $\stackrel{\infty}{-\infty}$ & $\stackrel{乛}{\xi}$ & $\underset{8}{8}$ & $\ddot{m}$ & $\stackrel{n}{n}$ & $\underset{\sigma}{\hat{\sigma}}$ & $\underset{i}{i}$ & $\stackrel{\sim}{n}$ & $\stackrel{\substack{n \\
i}}{i}$ & $\stackrel{m}{0}$ & $\stackrel{i}{i}$ & ส̇ & $\stackrel{?}{\stackrel{2}{2}}$ \\
\hline
\end{tabular}

Table 4: Table of Technical Attributes, with Final Weight and Mean, (Iqbal, et al., 2020)

\begin{tabular}{|c|c|c|c|c|c|c|c|c|c|c|c|c|c|c|}
\hline 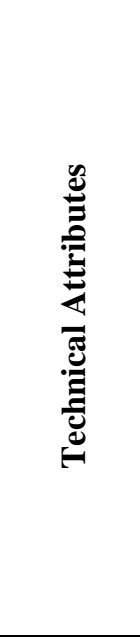 & 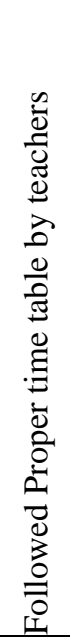 & 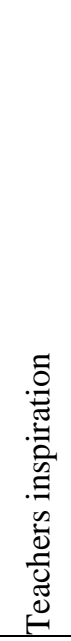 & 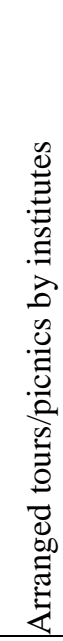 & 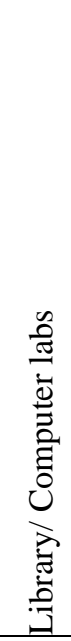 & 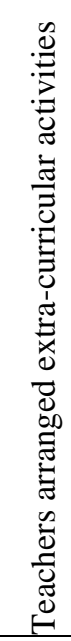 & 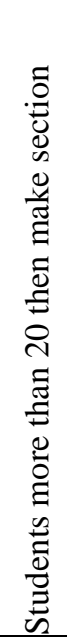 & 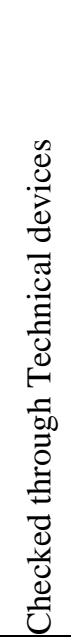 & 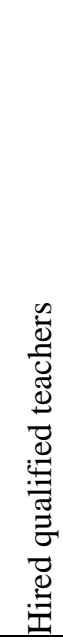 & 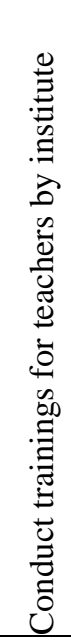 & 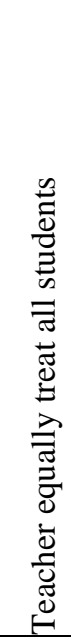 & 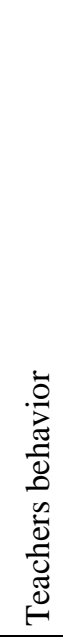 & 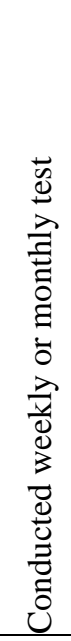 & 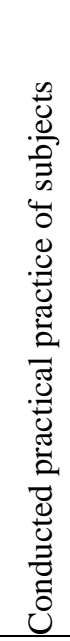 & 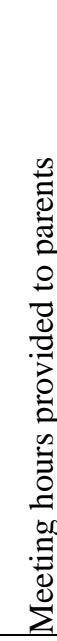 \\
\hline $\begin{array}{c}\text { Final } \\
\text { Weights }\end{array}$ & $\begin{array}{l}\infty \\
0 \\
i \\
i\end{array}$ & $\begin{array}{l}\stackrel{2}{i} \\
\text { ì }\end{array}$ & ণ্ণ & $\begin{array}{l}\infty \\
\stackrel{\infty}{i} \\
\ddot{\lambda}\end{array}$ & $\begin{array}{l}\overrightarrow{0} \\
\dot{q}\end{array}$ & $\begin{array}{l}\infty \\
\infty \\
0\end{array}$ & $\begin{array}{l}\text { ț } \\
\text { in }\end{array}$ & $=$ & $\begin{array}{l}\text { ọ } \\
\text { mे }\end{array}$ & 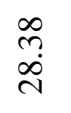 & 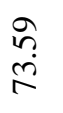 & 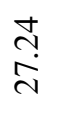 & $\begin{array}{l}5 \\
\dot{n} \\
m\end{array}$ & 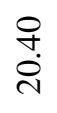 \\
\hline $\begin{array}{c}\text { Mean of } \\
\text { Final } \\
\text { Weights }\end{array}$ & $\hat{\sigma}$ & $\stackrel{\infty}{\infty}$ & $\stackrel{尺}{i}$ & $\underset{\text { dे }}{\text { in }}$ & $\stackrel{m}{m}$ & in & $\begin{array}{l}\hat{\sigma} \\
\dot{+}\end{array}$ & $\begin{array}{l}\hat{\sigma} \\
i\end{array}$ & $\begin{array}{l}\tilde{c} \\
\ddot{n}\end{array}$ & 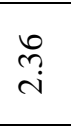 & $\frac{m}{6}$ & $\widehat{\widehat{N}}$ & $\hat{\widehat{\Lambda}}$ & $\stackrel{?}{I}$ \\
\hline
\end{tabular}

Using the methodology described above, we determine P-values. Table 5 and figure 2 express the P-values. 
Table 5: Table Showing, Final Weights of TAs, Means and P-values of the Case Study

\begin{tabular}{|c|c|c|c|c|c|c|c|c|c|c|c|c|c|c|}
\hline 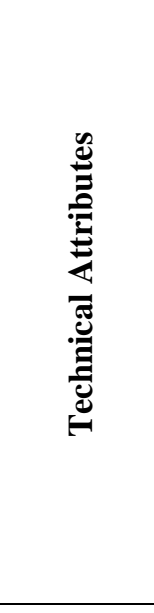 & 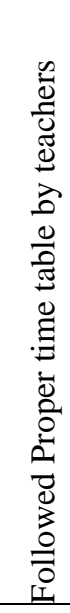 & 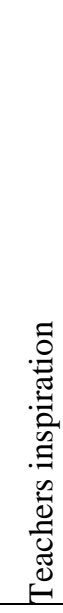 & 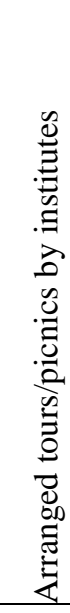 & 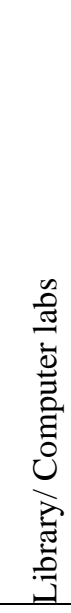 & 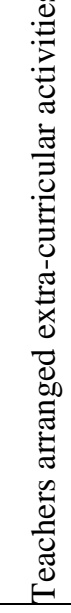 & 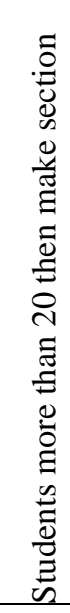 & 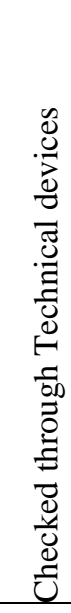 & 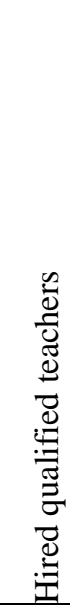 & 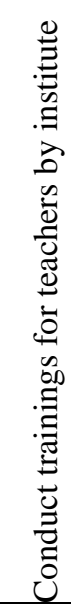 & 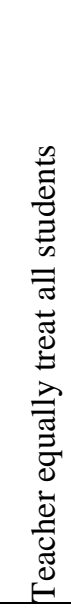 & 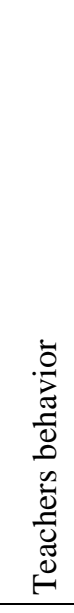 & 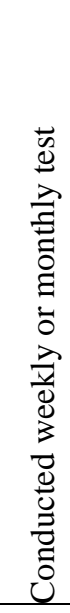 & 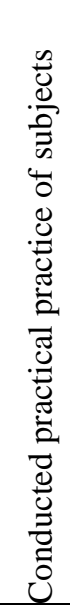 & 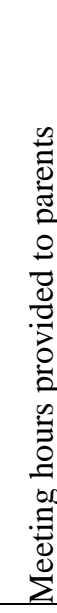 \\
\hline $\begin{array}{c}\text { Final } \\
\text { Weights }\end{array}$ & $\begin{array}{l}\infty \\
\stackrel{0}{0} \\
i\end{array}$ & 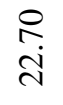 & 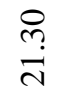 & 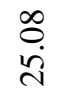 & 官 & $\begin{array}{l}\infty \\
\infty \\
\infty\end{array}$ & $\begin{array}{l}0 \\
\text { D. } \\
n\end{array}$ & $\vec{m}$ & के. & $\begin{array}{l}\infty \\
\stackrel{\infty}{0} \\
\infty \\
i\end{array}$ & ñ & 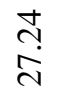 & $\begin{array}{l}\hat{0} \\
\dot{n}\end{array}$ & $\stackrel{?}{\stackrel{9}{0}}$ \\
\hline $\begin{array}{c}\text { Mean of } \\
\text { Final } \\
\text { Weights }\end{array}$ & $\stackrel{\hat{\sigma}}{+}$ & $\stackrel{\infty}{\infty}$ & $\stackrel{尺}{\stackrel{2}{2}}$ & $\underset{i}{i}$ & $\stackrel{m}{m}$ & $\stackrel{n}{6}$ & $\begin{array}{l}\hat{\sigma} \\
\dot{*}\end{array}$ & $\begin{array}{l}\hat{\sigma} \\
i\end{array}$ & $\stackrel{n}{n}$ & in & $\frac{9}{6}$ & $\hat{\sim}$ & $\widehat{\widehat{~}}$ & $\stackrel{?}{i}$ \\
\hline P-Values & 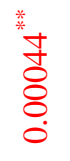 & 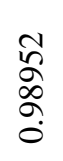 & ڤે & $\begin{array}{l}n \\
\infty \\
2 \\
\hat{o} \\
0\end{array}$ & 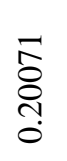 & $\begin{array}{l}\hat{\sigma} \\
\stackrel{\circ}{o} \\
\vdots \\
0\end{array}$ & 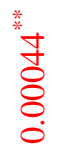 & 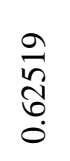 & 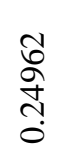 & $\begin{array}{l}\infty \\
\infty \\
\infty \\
\infty \\
\infty \\
0\end{array}$ & 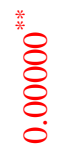 & 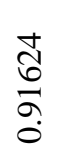 & $\frac{\infty}{\stackrel{\infty}{a}}$ & $\begin{array}{l}\frac{1}{0} \\
\stackrel{\circ}{\circ}\end{array}$ \\
\hline
\end{tabular}

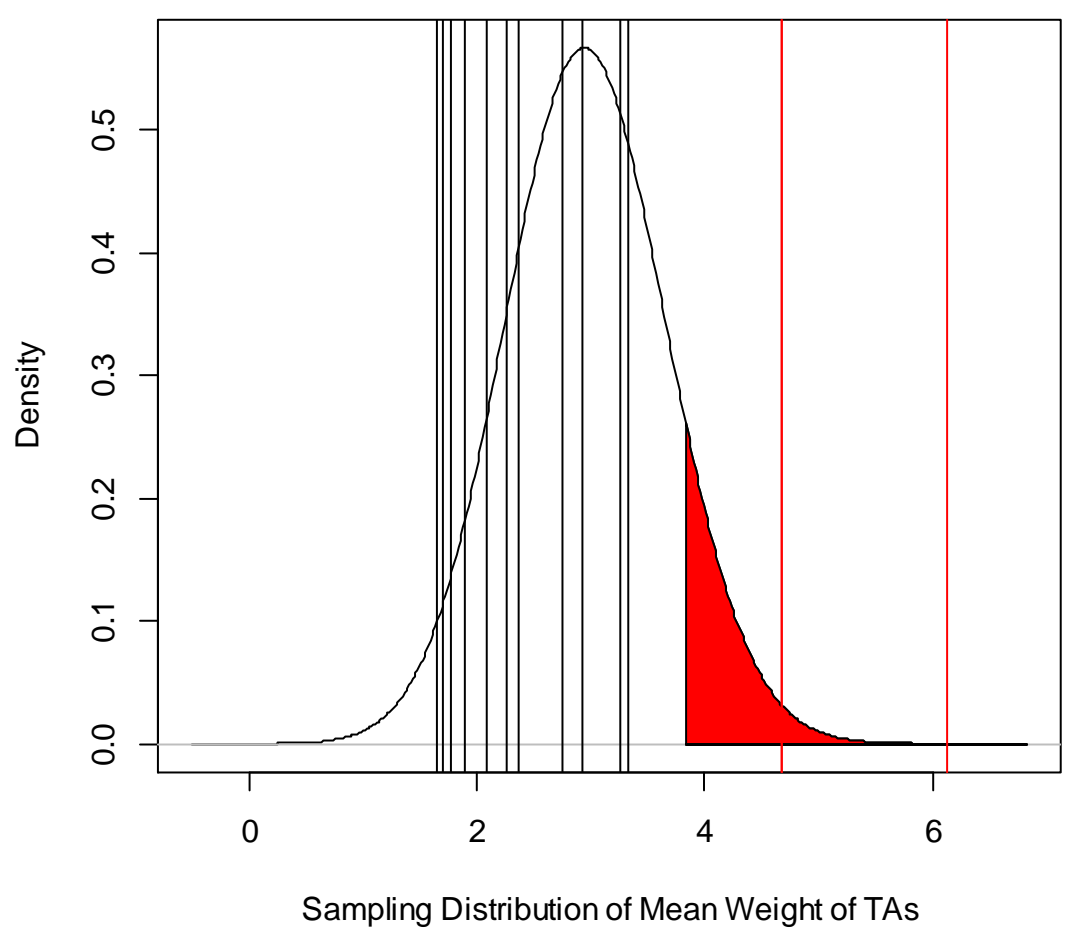

Figure 2: Density Plot Showing Significant TAs of Case Study 
Figure 2 shows our developed methodology, and concludes that in the case study there are three TAs which are found statistically significant. These are Teachers behavior, Followed Proper time table by teachers and Checked through Technical devices. These three TAs are statistically highly important with P-values, 0.0000. 0.0004 and 0.0004 respectively. As we see there are a total of twelve VOCs and fourteen TAs. The traditional QFD ranking method would have determined that Teachers behavior is the most important, and Followed Proper time table by teachers is the second most important. On the other end, Students more than 20 then make section is most least important and Meeting hours provided to parents is the second most least important. Over all, it is observed that out of fourteen TAs three have statistically significant importance from the remaining eleven 11 . We may say that roughly $20 \%$ are statistically significant, while $80 \%$ are non-significant. Describing differently, we may say that about $20 \%$ which statistically significant TAs, may resolve $80 \%$ of VOCs, while other $80 \%$ can only resolve $20 \%$ of VOCs. This situation in our case study shows an application of Pareto's 80/20 principle.

Now for further application of the developed methodology in other QFD case studies, we have selected five QFD case studies from articles published in international journals.

The first case study is taken from an article entitled "Using quality function deployment in manufacturing strategic planning (Crowe and Cheng, 1996). In this article the authors developed a new methodology for (manufacturing) strategic planning. In order to satisfy customer needs and demands, they selected fifteen TAs (Table 6) to improve manufacturing strategic planning. The current traditional way of QFD only provide the final weights of TAs, no further interpretation about the TAs is provided. Our developed methodology concluded that there are six TAs which are statistically significant, shown in red colour in table 6 and figure 3, while nine of them are statistically insignificant. Out of these six, four TAs i.e. A plant within a plant, Sell the Quality/Service, Buy substitute products and resell, Instill Customer first attitude, are determined to be statistically highly significant, with P-values, 0.0022, 0.0016, 0.0010, 0.0000 respectively. While two i.e. Adopt Cellular Approach and Focus on small order are proved as significant, i.e., with P-values, 0.0213, 0.0213 respectively. Figure 3 red lines represents the significance of TAs, while black lines express insignificance of TAs.

Table 6: Final Weights of TAs, Means and P-values of the case study, (Crowe and Cheng, 1996)

\begin{tabular}{|c|c|c|c|c|c|c|c|c|c|c|c|c|c|c|c|}
\hline 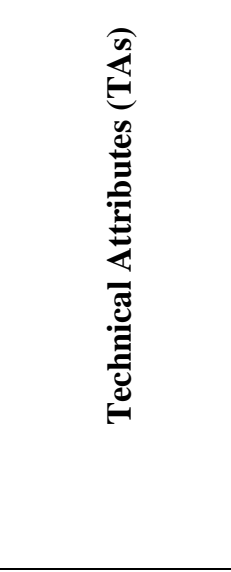 & 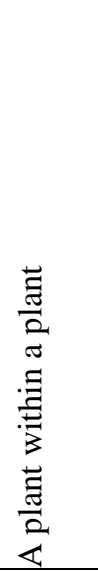 & 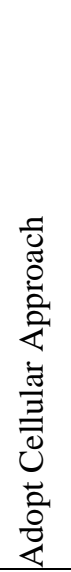 & 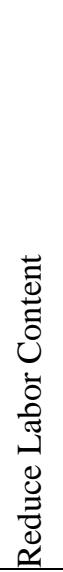 & 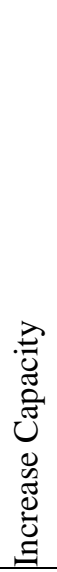 & 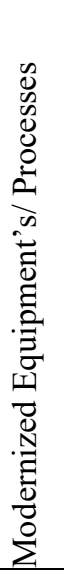 & 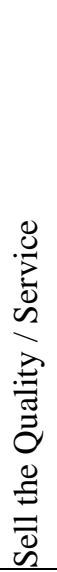 & 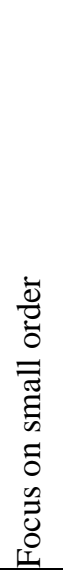 & 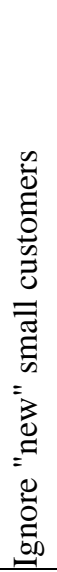 & 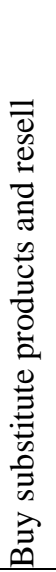 & 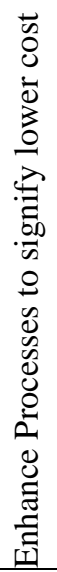 & 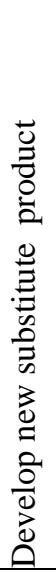 & 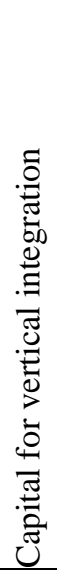 & 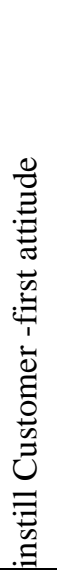 & 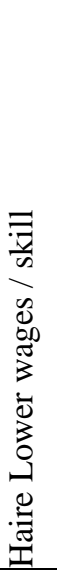 & 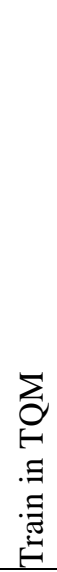 \\
\hline Final Weights & 87 & 80 & 32 & 36 & 52 & 88 & 80 & 56 & 89 & 65 & 62 & 39 & 95 & 20 & 75 \\
\hline $\begin{array}{l}\text { Mean of Final } \\
\text { Weights }\end{array}$ & $\begin{array}{l}\infty \\
\infty \\
n\end{array}$ & $\stackrel{m}{m}$ & $\stackrel{m}{m}$ & 6 & $\vec{\infty}$ & 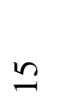 & 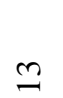 & $\dddot{m}$ & $\begin{array}{l}\infty \\
\infty \\
\dot{I}\end{array}$ & $\stackrel{\infty}{\infty}$ & 으 & ? & $\simeq$ & $\stackrel{m}{m}$ & $\stackrel{2}{2}$ \\
\hline
\end{tabular}




\begin{tabular}{|c|c|c|c|c|c|c|c|c|c|c|c|c|c|c|c|}
\hline P-Values & 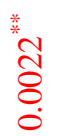 & 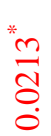 & $\begin{array}{l}8 \\
8 \\
8\end{array}$ & $\begin{array}{l}\text { ลे } \\
\text { Һे }\end{array}$ & $\begin{array}{l}\overline{\widehat{N}} \\
\text { } \\
\hat{0}\end{array}$ & $\frac{\text { *o }}{\frac{*}{8}}$ & 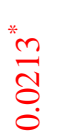 & $\begin{array}{l}8 \\
8 \\
\infty \\
0 \\
0\end{array}$ & $\begin{array}{l}\stackrel{*}{\circ} \\
\stackrel{\circ}{\circ} \\
\stackrel{0}{\circ}\end{array}$ & 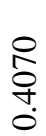 & 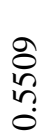 & $\begin{array}{l}\hat{\sigma} \\
\text { }\end{array}$ & 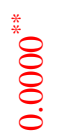 & $\begin{array}{l}8 \\
8 \\
8\end{array}$ & 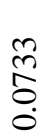 \\
\hline
\end{tabular}

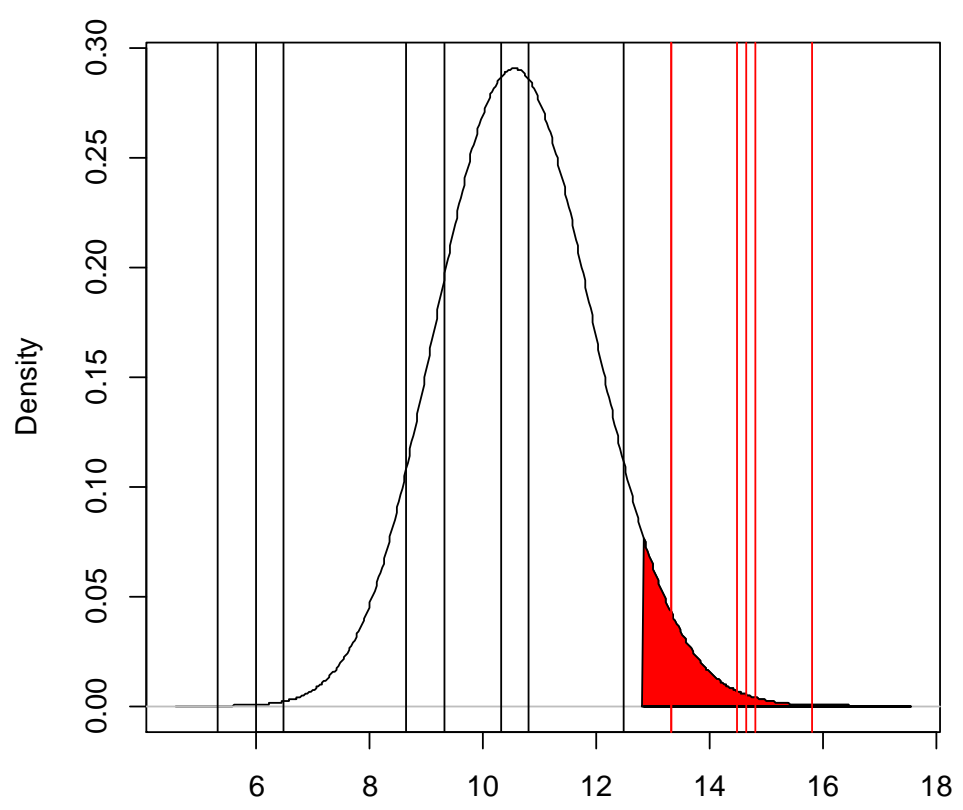

Sampling Distribution of Mean Weight of TAs, Crowe \& Cheng, 1996

Figure 3: Density plot showing significance of TAs, (Crowe and Cheng, 1996)

The second case study is taken from published article entitled "Integrated design and production of multi-story timber frame houses - production effects caused by customer-oriented design" (Stehn and Bergström, 2002). In this, there are a total of six TAs suggested for multi-storey timber frame houses. The final weights of these six TAs suggest nothing except the ranking on the basis of FWs. Our developed methodology determined that out of these six TAs, three, i,e, Material choice, and Integrated design production proved highly significant with P-values 0.0000 and 0.0000 respectively, while Visible Technical Installation is demonstrated of significant importance with P-value 0.0333. Table 7 and figure 4 shows the P-values in red text and red line.

Table 7: Final Weights of TAs, Means and P-values of the Case Study, (Stehn and Bergström, 2002)

\begin{tabular}{|c|c|c|c|c|c|c|}
\hline $\begin{array}{c}\text { Technical Attributes, } \\
\text { (TAs) }\end{array}$ & 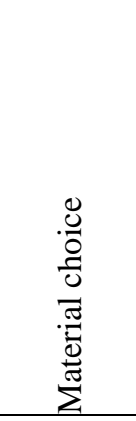 & 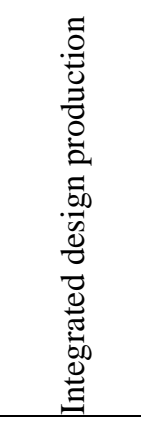 & 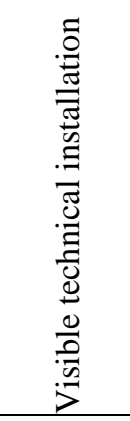 & 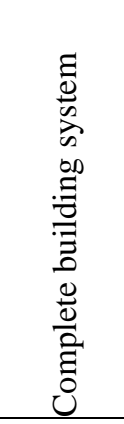 & 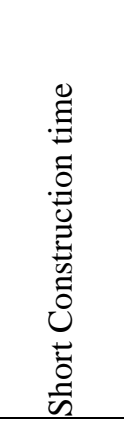 & 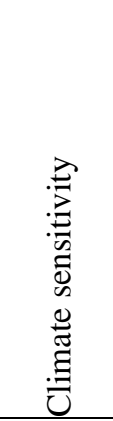 \\
\hline Final Weights & 213 & 235 & 172 & 118 & 90 & 70 \\
\hline Mean of Final Weights & 23.70 & 26.00 & 19.10 & 13.10 & 10.00 & 7.80 \\
\hline P-Values & $0.0000^{* *}$ & $0.0000^{* *}$ & $0.0333^{*}$ & 0.9958 & 1.0000 & 1.0000 \\
\hline
\end{tabular}




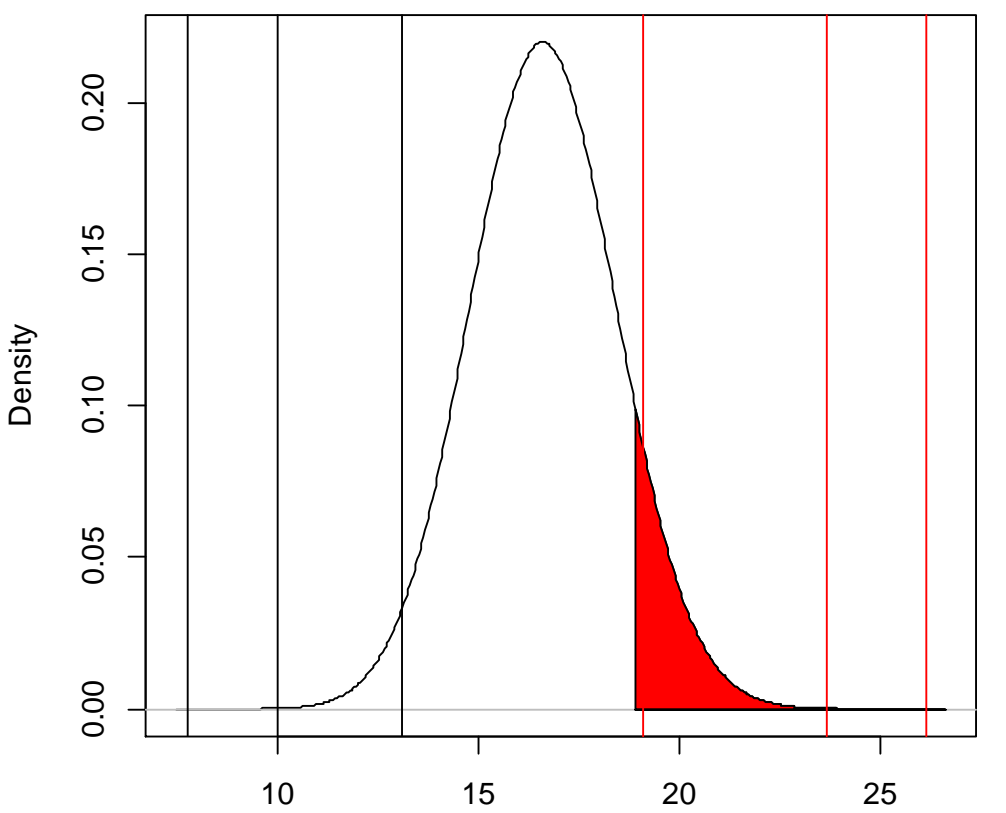

Sampling Distribution of Mean Weight of TAs, Stehn \& Bergström, 2002

Figure 4: Density Plot Showing Significance of TAs, (Stehn and Bergström, 2002)

The third case study is taken from the article, entitled "Product attribute function deployment (PAFD) for decision-based conceptual design" (Hoyle and Chen, 2009). Current final results of case study express final weights along with their ranking. Our developed methodology determined that out of six two TAs are of highly significant importance. Table 8 and figure 5 shows the P-values in red text and red line.

Table 8: Final Weights of TAs, Means and P-values of the Case Study, (Hoyle and Chen, 2009)

\begin{tabular}{|c|c|c|c|c|c|c|}
\hline Technical Attributes & 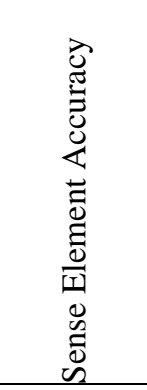 & 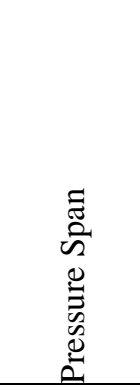 & 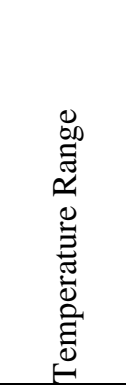 & 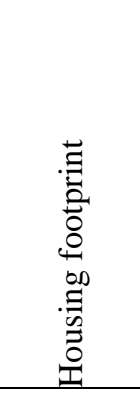 & 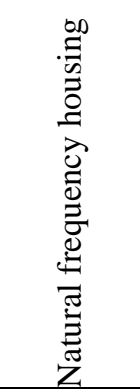 & 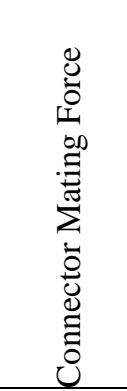 \\
\hline Final Weights & 72 & 82 & 58 & 48 & 36 & 26 \\
\hline Mean of Final Weights & 12.00 & 13.67 & 9.67 & 8.00 & 6.00 & 4.30 \\
\hline P-Values & $0.00716^{*}$ & $0.00015^{*}$ & 0.25125 & 0.75529 & 0.99298 & 0.99999 \\
\hline
\end{tabular}




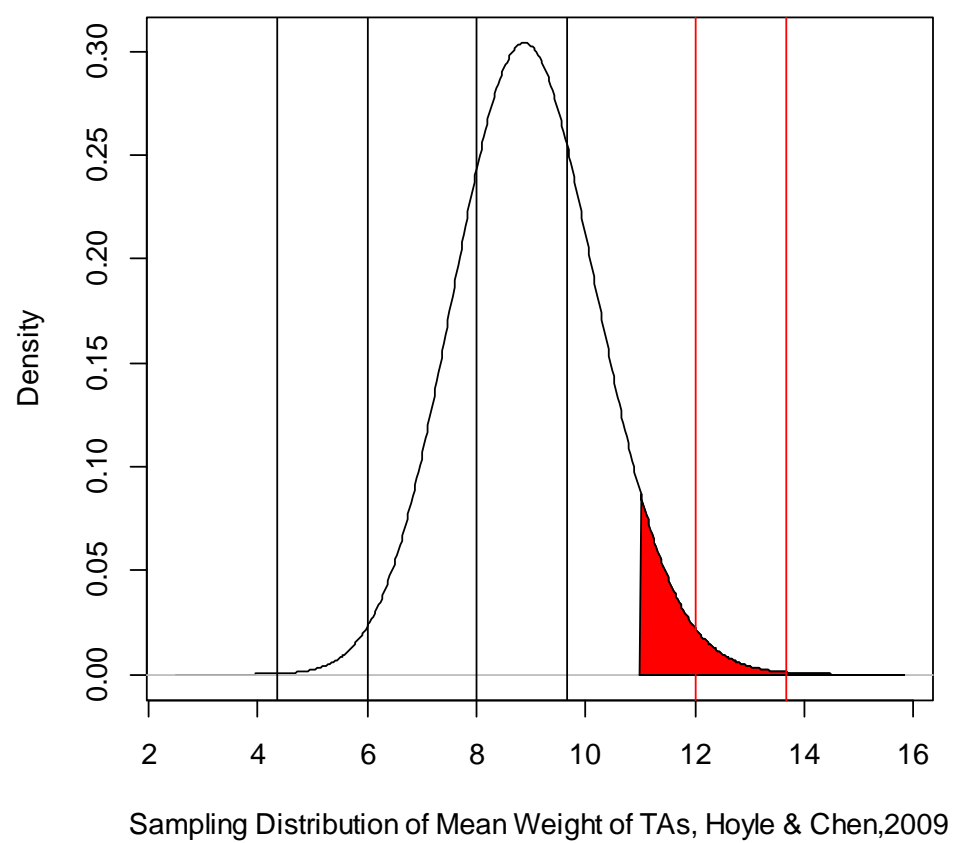

Figure 5: Density plot Showing Significance of TAs, (Hoyle and Chen, 2009)

Case studies 4 and 5 shown in appendices I and II, respectively.

In Case study 4 (Appendix I), QFD is used to prioritize National Board of Accreditation (NBA) quality parameters in engineering education in to order to satisfy student's needs and demands, (Sing and Rawani, 2019). Current final results of case study express final weights of TAs and ranks of TAs. The P-values determined (Table AI, Figure AI) express three prominent TAs are, Student Performance, Facilities \& Technical Support and Improvement attainment outcomes with P-values, 0.00, 0.03 and 0.00. Its shows the maximum student's satisfaction can be achieved through the three TAs.

In Case study 5 (Appendix II), taken from the article, entitled "Linking operation plans to business objectives using QFD" (Dror, 2019) the author utilises QFD method for ranking the operation plans of an individual organisation to achieve business objectives. Our developed methodology shows that Stable Product Quality is the only significant TA, having P-value 0.0114, (Table AII, Figure AII).

\section{Discussion}

Regarding to the main objective of this research, we have argued that current QFD practice only determines final weights of TAs and sets a priority order for resolution. Our developed methodology supports our proposition that the final weights may be categorized into significant and insignificant final weights. We have developed a new methodology to show that how the significance of the mean weights of TAs can be determined. We employed the developed methodology on our conducted case study, as a real-life example and also we employed the methodology on some published case studies taken from the international research articles. The P-values derived as last important step from the actual mean weights of TAs, demonstrate that not all of the mean weights are statistically equally important.

The finding from the above results express that TAs statistically may be divided into two groups, i.e. group of insignificant TAs and group of significant TAs. These groups have homogeneity (internal consistency) within the groups but heterogeneity between the groups. The Pareto principle (the 80/20 rule) in general supports our contention that significant TAs are those which contribute the maximum to customer satisfaction, and should be resolved on a priority basis. In 
the case of selected case studies, we observe not all the TAs significant or insignificant, but some case study, it might happen that all of the TAs determined to be insignificant or vice versa. The developed methodology provide a statistical way for better determination of TAs, although, practitioners/engineers may choose those TAs which are necessary to maximise customer satisfaction in the development of a QFD analysis. The literature review shows that there is lot of variability in the ordinal rating in form of length, strength from matrix to matrix. So, for the selection of measure of central tendency, practitioners should be careful. A good selection of central value helps to simulate a better hypothetical population. Practitioner/researchers should also be careful in the use of level of significance. The most commonly used QFD implementation ignores the integration of factors such as cost, the length of the project to complete, life cycle, and available resources.

\section{Conclusion}

Core principles of quality management include

- Decision making based on facts, data or evidence

- Understanding variation, and separating common from special cause variation, and

- Continuous improvement of systems and methods.

In this paper, we identify a potential weakness of the QFD methodology, effective and popular though it is. We apply the general principle of separating common from special cause variation to introduce a more statistically reliable (significance-based) method of determining which TAs should be prioritized for incorporation into the product or process design. In doing so, we introduce a more fact-based decision-making basis, and improve the tested methodology of QFD.

A practically applied consequence of this methodology is that institutions/organisations may occupy in costly and time taking activities as a resulting from the high preference of statistical significant TAs, on the other hand an attribute requiring less effort or cost may be an equal order as insignificant TAs. However, in some cases a small difference in P-values may not clearly defined, which TA should resolve on priority basis and which to resolve with convenience.

On the other hand, the final results of TAs be adopted by managerial, administrative or consultant practitioners. But they should be aware of their applied consequences and constraints. In case of such implications, it is suggested to discuss with TAs expert team. All the stake holders can sit for strategic planning and get advice from someone knowledgeable about the quality tools.

\section{References}

Ahmed, S. (2006), "QFD Application to improve management education at KIMEP" Issues in Information Systems, Vol. VII No. 1, pp. 193-198.

Andronikidis, A., Georgiou, A.C., Gotzamani, K. and Kamvysi, K. (2009), "The application of quality function deployment in service quality management" The TQM Journal, Vol. 21 No. 4, pp. 319-333.

Aytac, A. and DENIZ, V. (2005), "Quality Function Deployment in Education: A Curriculum Review." Quality \& Quantity, Vol. 39, pp. 507-514.

Barnett, W. D. and Raja, M. K. (1995). "Application of QFD to the software development process." International Journal of Quality \& Reliability Management, Vol. 12, No.6, pp. 24-42.

Benner, M., Linnemann, A. R., Jongena, W. M. F. and Folstara, P. (2003), "Quality Function Deployment (QFD) - can it be used to develop food products?" Food Quality and Preference, Vol. 14, pp. 327-339.

Chang, C.-L. (2006), "Application of quality function deployment launches to enhancing nursing home service quality." Total Quality Management \& Business Excellence, Vol. 17 No. 3, 
pp. 287-302.

Chin, K.-S., Pun, K.-F., Leung, W. M. and Lau, H. (2001), "A quality function deployment approach for improving technical library and information services: a case study." Library Management, Vol. 22 No. 4/5, pp. 195-204.

Crowe, T. J. and Cheng, C.-C. (1996), "Using quality function deployment in manufacturing strategic planning." International Journal of Operations \& Production Management, Vol. 16 No.4, pp. 35-48.

Einspruch, E. M., Omachonu, V. K. and Einspruch, N. G. (1996), "Quality function deployment (QFD): application to rehabilitation services." International Journal of Health Care Quality Assurance, Vol. 9 No. 3, pp. 41-46.

Esteban-Ferrer, M. J. and Tricás, J. (2012), "Applying QFD to strategic quality management in law firms." Total Quality Management \& Business Excellence, Vol. 23 No. 12, pp. 14331451.

Franceschini, F. and Rossetto, S. (2002), “QFD: an interactive algorithm for the prioritization of product's technical design characteristics", Integrated Manufacturing Systems, Vol. 13 No.1, pp. 69-75.

Gambarov, V., Sarno, D., Hysa, X., Calabrese, M. and Bilotta, A. (2017), "The role of loyalty programs inhealth care service ecosystems" The TQM Journal, Vol. 29 No. 6, pp. 899919

Gonzalez, M. E., Quesada, G., Picado, F. and Eckelman, C. A. (2004), "Customer satisfaction using QFD: an e-banking case." Managing Service Quality, Vol. 14, No.4, pp. 317-330.

Halbleib, L. (1993), "Application of Quality Function Deployment to the Design of a Lithium Battery." IEEE Xplore, pp. 150-154.

Harber, N and Fargnoli, M. (2019). "Prioritizing customer requirements in a product-service system(PSS) context." The TQM Journal, Vol. 31 No.2 pp. 257-273.

Herrmann, A., Huber, F., Algesheime, R. and Tomczak, T. (2006). "An empirical study of quality function deployment on company performance" International Journal of Quality \& Reliability Management Vol. 23 No.4, pp. 345-366.

Hoyle, C. J. and Chen, W. (2009), "Product Attribute Function Deployment (PAFD) for Decision-Based Conceptual Design." IEEE TRANSACTIONS ON ENGINEERING MANAGEMENT, Vol. 56 No. 2, pp. 271-284.

Iqbal, Z., Grigg, N. P., Govindaraju, K. and Campbell-Allen, N. (2014), "Statistical comparison of final weight scores in quality function deployment (QFD) studies." International Journal of Quality \& Reliability Management, Vol. 31 No. 2, pp. 184-204.

Iqbal, Z. and Grigg, N.P. (2020), "Enhancing voice of customer prioritisation in QFD by integrating the competitor matrix", International Journal of Productivity and Performance Management, Vol. ahead-of-print No. ahead-of-print. https://doi.org/10.1108/IJPPM-042019-0175.

Jeong, M. and Oh, H. (1998), "Quality function deployment: An extended framework for service quality and customer satisfaction in the hospitality industry." Hospitality Management, Vol. 17, pp. 375-390.

Lam, K. and Zhao, X. (1998), "An application of quality function deployment to improve the quality of teaching." International Journal of Quality \& Reliability Management, Vol. 15 No.4, pp. 389-413.

Laosirihongthong, T., Adebanjo, D., Samaranyake, P., Subramanian, N. (2018), "Prioritizing warehouse performance measures in contemporary supply chains.", International Journal of Productivity and Performance Management, Vol. 67 No. 9, pp. 1703-1726

Maritan, D., and Panizzolo, R. (2009). "Identifying business priorities through quality function deployment: Insights from a case study." Marketing Intelligence \& Planning, 27(5), 714728.

Masuri, K., Sakao, T., Kobayashi, M. and Inaba, A. (2003) "Applying Quality Function Deployment to environmentally conscious design." International Journal of Quality \& 
Reliability Management, Vol. 20 No.1, pp. 90-106.

Mazur, G. H. (1993), "QFD for Service Industries." Japan Business Consultants. Ltd, pp. 1-17.

Olewnik, A. and Lewis, K. (2008), "Limitations of the House of Quality to provide quantitative design information" International Journal of Quality \& Reliability Management, Vol. 25 No. 2, pp. 125 - 146.

Paryani, K., Masoudi, A. and Cudney, E. (2010), "QFD Application in the Hospitality Industry: A Hotel Case Study." American Society for Quality, Vol. 17 No. 1, pp. 7-28.

Partovi, F. Y. and Corredoira, R. A. (2002), "Quality function deployment for the good of soccer." European Journal of Operational Research, Vol. 137, pp. 642-656.

Raharjo, H., Xie, M., Goh, T. N. and Brombacher, A. C. (2007), "A Methodology to Improve Higher Education Quality using the Quality Function Deployment and Analytic Hierarchy Process." Total Quality Management \& Business Excellence, Vol. 18 No.10, pp. 1097-1115.

Rahim, A. R. A. and Baksh, M. S. N. (2003), "Application of Quality Function Deployment (QFD) method for Pultrusion machine design planning" Industrial Management \& Data Systems, Vol. 103 No. 6, pp. 373-387.

Sing, A. K and Rawani, A.M. (2019), "Application of quality function deployment for the prioritization of National Board of Accreditation quality parameters" Quality Assurance in Eduction, Vol. 27 No. 1, pp. 127-139

Snee, R.D. (1990), Source: The American Statistician, Vol. 44, No. 2 (May, 1990), pp. 116-121

Stehn, L. and Bergstrom, M. (2002), "Integrated design and production of multi-storey timber frame houses - production effects caused by customer-oriented design." Int. J. Production Economics, Vol. 77, pp. 259-269.

Tan, K., Xie, M. and Chia, E. (1998), "Quality function deployment and its use in designing information technology systems" International Journal of Quality \& Reliability Management, Vol. 15 No.6, pp. 634-645.

TAN, B. L., TANG, N. K. H. and FORRESTER P. L. (2004), "Application of QFD for eBusiness planning." Production Planning \& Control: The Management of Operations, Vol. 15 No. 8, pp. 802-818.

Thakkar, J., Deshmukh, S. and Shastree, A. (2006), "Total quality management (TQM) in selffinanced technical institutions: a quality function deployment (QFD) and force field analysis approach" Quality Assurance in Education, Vol. 14 No. 1, pp. 54-74.

Tortorella, G., Cauchick-Miguel, P. A. and Gaiardelli, P. (2018), "Hoshin Kanri and A3: $a$ proposalfor integrating variability into thepolicy deployment process" The TQM Journal, Vol. 31 No. 2, pp. 118-135.

Trappey, C. V., Trappey, A. J. C. and Hwang, S-J (1996), "A COMPUTERIZED QUALITY FUNCTION DEPLOYMENT APPROACH FOR RETAIL SERVICES." Computers ind. Engng, Vol. 30 No. 4, pp. 611-622.

Tusnial, A., Sharma, S.K., Dhingra, P. and Routroy, S. (2020), "Supplier selection using hybrid multicriteria decision-making methods", International Journal of Productivity and Performance Management, Vol. ahead-of-print No. ahead-of-print. https://doi.org/10.1108/IJPPM-04-2019-0180

Viaene, J. and Januszewska, R. (1999), "Quality function deployment in the chocolate industry." Food Quality and Preference, Vol. 10 No. 10, pp. 377-385.

Vinodh, S. and Chintha, S. K. (2011), "Application of fuzzy QFD for enabling leanness in a manufacturing organization." International Journal of Production Research, Vol. 49 No. 6, pp. 1627-1644.

Walker, M. (2002), "Customer-driven break through using QFD and policy deployment." Management Decision, Vol. 40 No.3, pp. 248-256.

Wu, H.-Y. and Lin, H.-Y. (2012), "A hybrid approach to develop an analytical model for enhancing the service quality of e-learning." Computers and Education, Vol. 58, pp. 1318-1338. 
Yamane, T. (1967), Statistics, An Introductory Analysis, 2nd Ed., New York: Harper and Row.

\section{Appendix AI:}

Table AI: Final Weights of TAs, Means and P-values of the case study, (Sing and Rawani, 2018)

\begin{tabular}{|c|c|c|c|c|c|c|c|c|c|}
\hline & 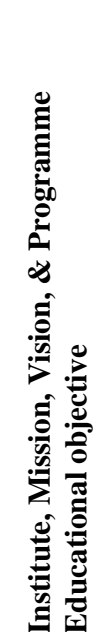 & 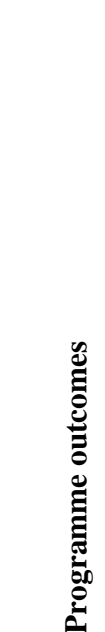 & 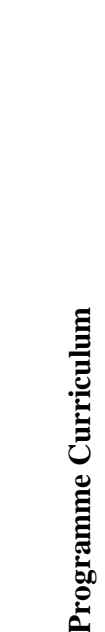 & 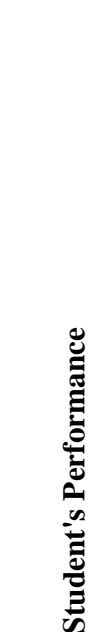 & 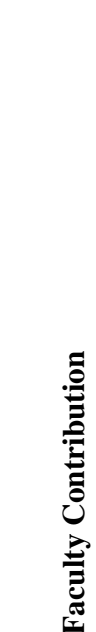 & 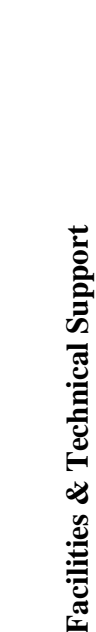 & 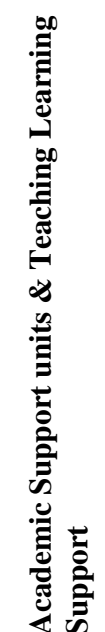 & 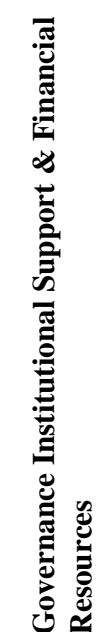 & 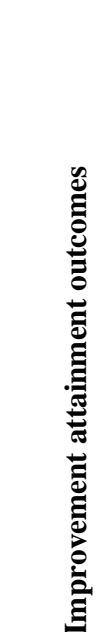 \\
\hline Final Weights & 1327 & 1335 & 1170 & 1561 & 1226 & 1398 & 1260 & 1299 & 1497 \\
\hline Mean of Final Weights & 26.02 & 26.18 & 22.94 & 30.61 & 24.04 & 27.41 & 24.71 & 25.47 & 29.35 \\
\hline P-Values & 0.64 & 0.56 & 1.00 & 0.00 & 0.99 & 0.03 & 0.99 & 0.87 & 0.00 \\
\hline
\end{tabular}

Figure AI: Density plot Showing significance of TAs, (Sing and Rawani, 2018)

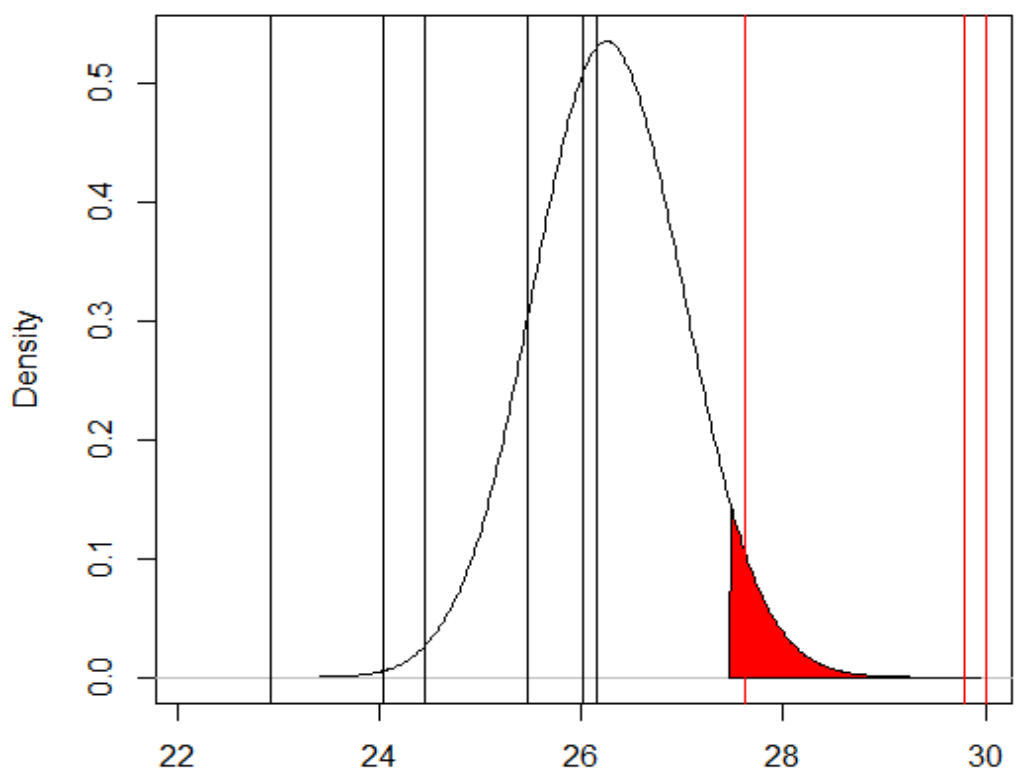

Sampling Distribution of Mean Weight of TAs, Sing and Rawani, (2018)

\section{Appendix AII:}

Table AII: Final Weights of TAs, Means and P-values of the case study, (Dror, 2019) 


\begin{tabular}{|c|c|c|c|c|c|c|c|}
\hline 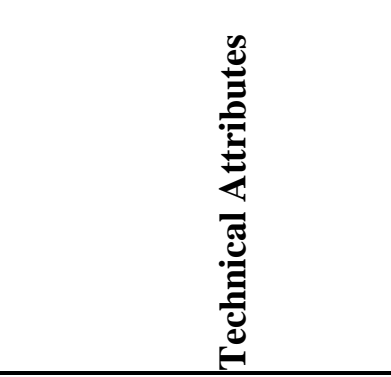 & 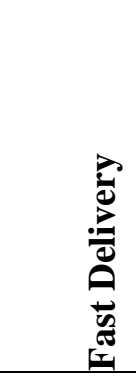 & 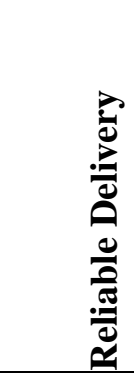 & $\begin{array}{l}\overrightarrow{0} \\
\dot{\theta} \\
\dot{\theta}\end{array}$ & 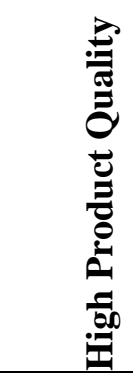 & 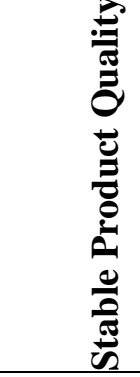 & 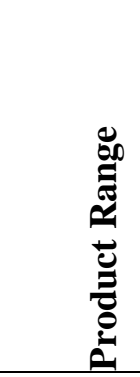 & 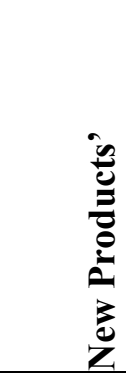 \\
\hline Final Weights & 5.46 & 5.88 & 1.44 & 1.28 & 8.16 & 1.44 & 1.44 \\
\hline Mean of Final Weights & 1.82 & 1.96 & 0.48 & 0.43 & 2.72 & 0.48 & 0.48 \\
\hline P-Values & 0.1577 & 0.2981 & 0.7026 & 0.8786 & 0.0114 & 0.8786 & 0.8786 \\
\hline
\end{tabular}

Figure AII: Density plot Showing significance of TAs, (Dror, 2019)

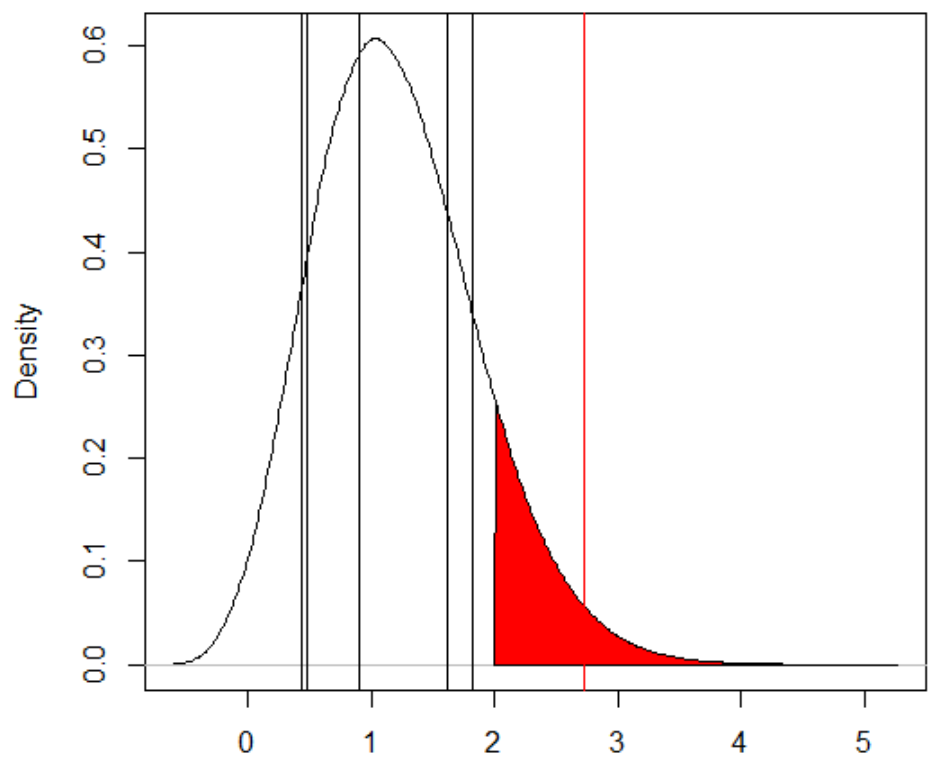

Sampling Distribution of Mean Weight of TAs, Dror, 2019. 